\title{
Collective Value Creation and Empowerment in an Online Brand Community: A Netnographic Study on LEGO Builders
}

\author{
Hanna Kurikko and Pekka Tuominen
}

\author{
"Online communities are not virtual. The people that we" \\ meet online are not virtual. They are real communities \\ populated with real people, which is why so many end \\ up meeting in the flesh. \\ Robert Kozinets \\ Professor of Marketing \\ Author of Netnography (tinyurl.com/7eoklae)
}

\begin{abstract}
Online communities are becoming "places" of belonging, information, and emotional support that people cannot do without. These social groups have a real existence for their participants, and thus have consequential effects on many aspects of behaviour. This article examines collective value creation and empowerment in an online brand community. It presents the main features of an online brand community, the process of value co-creation, and motivators for participating in online brand communities. These key factors jointly characterize collective value creation and empowerment. This netnographic study focuses on an online brand community called BrickBuilders, which is a meeting place for LEGO builders in Finland. BrickBuilders' members feel a sense of belonging, they share similar motivations, and they create value together.
\end{abstract}

\section{Introduction}

A brand community can be formed by any group of people who share a common interest in a specific brand and who create a parallel social universe rife with its own myths, values, rituals, vocabulary, and hierarchy (Muniz and O'Guinn, 2001: tinyurl.com/d73sov4; Cova and Pace, 2006: tinyurl.com/ccp6jy3). Brand communities become more than a place. They become a common understanding of a shared identity, which can be found in both face-to-face interactions and in cyberspace (Muniz and O'Guinn, 2001).

Analysts no longer question whether the concept of community should have a place in the domain of marketing (Cova and Pace, 2006). However, the concepts of brand community and online brand community are relatively new and have yet to find their place in the academic world.
Traditionally, companies produced products relatively independently. Today, consumers and other stakeholders can create value more collectively. The purpose of this article is to describe and analyze collective value creation and empowerment in an online brand community.

\section{Main Features of Online Brand Communities}

Muniz and O'Guinn (2001; tinyurl.com/d73sov4) used three constructs to identify the distinguishing features of brand communities. First, a sense of belonging is a connection that members feel toward one another and the collective sense of difference from others outside of the community.

The second feature is the presence of shared rituals and traditions that surround the brand. Rituals and traditions perpetuate the community's shared history, cul- 


\section{Collective Value Creation and Empowerment in an Online Brand Community}

Hanna Kurikko and Pekka Tuominen

ture, and consciousness. Traditions include certain behavioural norms and values.

The third feature is a sense of moral responsibility, which is a felt sense of duty or obligation to the community. The sense of moral responsibility is what produces collective action.

Heinonen and Halonen (2007; tinyurl.com/cl8qwwb) have identified motivators for online brand community activities. Members want to belong to something, build and strengthen their identities, get feedback from others, and create something new.

\section{The Process of Collective Value Creation}

Schau and colleagues (2009; tinyurl.com/bsh984y) have identified the process of value co-creation in online brand communities. The process consists of four thematic practices, which are social networking, impression management, community engagement, and brand use.

Social networking is a practice that focuses on creating, enhancing, and sustaining ties among brand community members. These include welcoming, empathizing, and governing. These practices operate primarily in the intangible domain of the emotions and reinforce the social or moral bonds within the community.

Impression management includes evangelizing and justifying. Online brand community members act as altruistic emissaries and ambassadors of good will. Members devote time and effort to the brand, share the news of the brand, and inspire others to participate in the community.

Community-engagement practices are those that reinforce members' escalating engagement with the brand community. These include staking, milestoning, badging, and documenting. Staking, milestoning, and badging mean that community members bring out brand experiences and proclaim openly that they are fans of a particular brand. Documenting occurs when brand community members construct a narrative of their brand experiences.

Brand-use practices are specifically related to improved or enhanced use of the focal brand. These include grooming, customizing, and commoditizing. Grooming means that members share, for example, homemade tools and advice. Customizing means modifying existing ideas and discovering new ideas, which result in customized products. Commoditizing means that members rant or chastise some products, but at the same time, they have new ideas on how those products could be developed.

\section{Synthesis of the Theoretical Framework}

The main features of online brand communities, value co-creation, and motivators for participating in online brand communities (Heinonen and Halonen, 2007: tinyurl.com/cl8qwwb; Kozinets, 2010: tinyurl.com/7eoklae; Muniz and O'Guinn, 2001: tinyurl.com/d73sov4; Schau et al., 2009: tinyurl.com/bsh984y) are the key factors that jointly - realized in various combinations - characterize collective value creation and empowerment in an online brand community. The collective value creation and empowerment in the online brand community may occur when its members have a sense of belonging, they create value together, and they have similar motives.

The collective value creation and empowerment of the online brand community allows mutual interaction between the online brand community and the company as well as other stakeholders. Companies have an opportunity to communicate with consumers and influence their opinions (Kozinets, 2010; tinyurl.com/7eoklae) and vice-versa. We have moved away from one-way transactions to a relationship-based interaction model that emphasizes consumers' and other stakeholders' roles in networks and communities. Figure 1 illustrates the process of collective value creation and empowerment in an online brand community. This provides a theoretical framework for this study.

\section{Conducting the Netnographic Study}

Netnography is "a new qualitative research methodology that adapts ethnographic research techniques to study cultures and communities that are emerging through computer-mediated communications" (Kozinets, 2002; tinyurl.com/7sb92xl). The netnography method is about observing participants in online contexts (Kozinets, 2010: tinyurl.com/7eoklae; Rokka, 2010: tinyurl.com/ $6 \mathrm{v} 7 \mathrm{~b} 4 \mathrm{sn})$. Some of the most important standards of quality in netnography are immersive depth, prolonged engagement, researcher identification, and persistent conversations. (Kozinets, 2006: tinyurl.com/7j92tjp; Muniz and Schau, 2007: tinyurl.com/84kudob). 


\section{Collective Value Creation and Empowerment in an Online Brand Community}

\section{Hanna Kurikko and Pekka Tuominen}

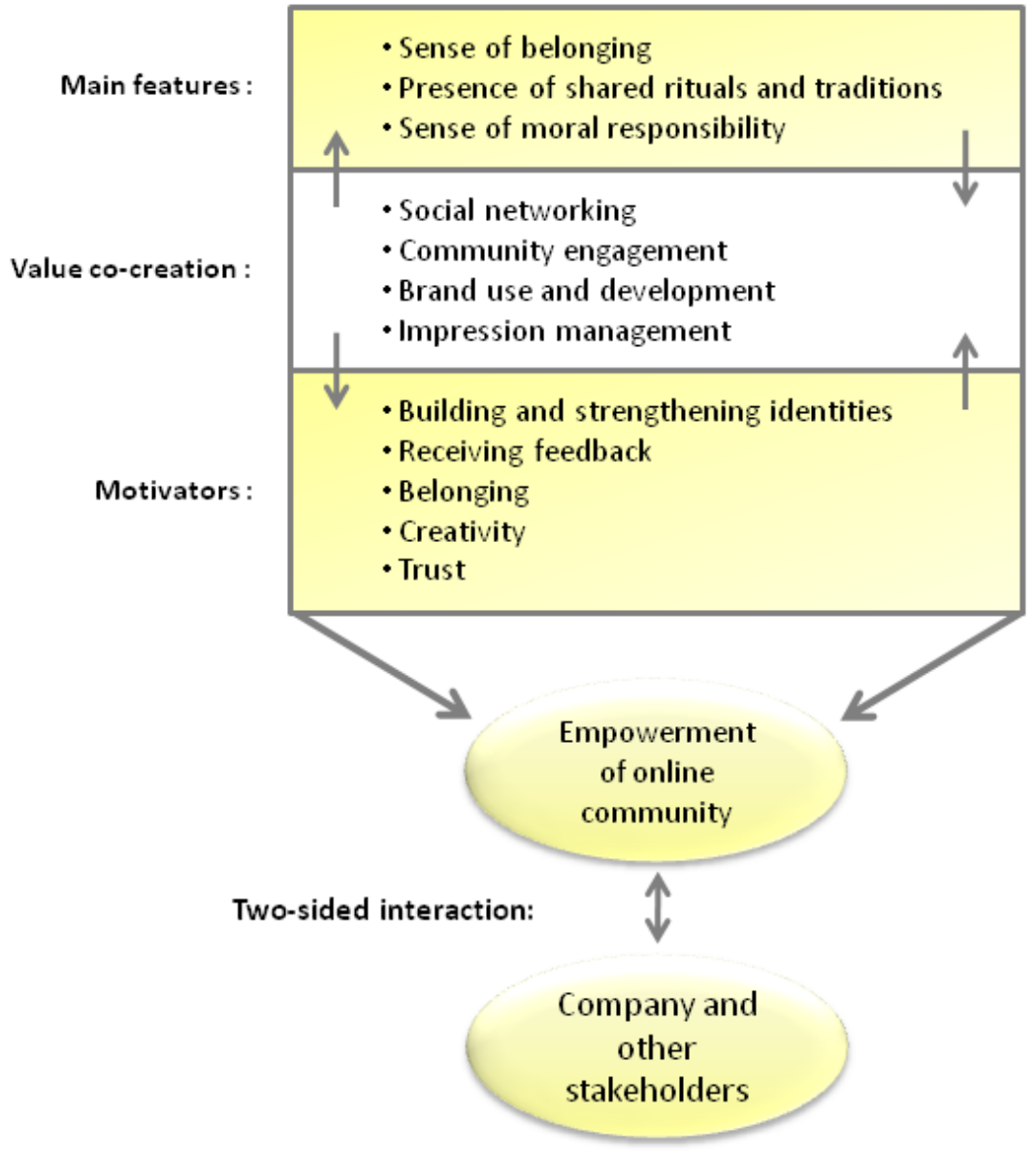

Figure 1. The process of collective value creation and empowerment of an online brand community

In this study, the object is an active online brand community called BrickBuilders (Palikkatakomo; palikkatakomo.org). It is a meeting place for LEGO builders in Finland. The community is fully fan-based and is not sponsored by the LEGO company. As of April 2011, BrickBuilders had 350 members. The community's website had over 12,000 postings, almost 900 threads, and 10 different subject categories in the forum.

BrickBuilders was observed from November 2010 to the end of April 2011. In this study, 1,035 different postings that contained 197 distinct poster names were analyzed. The researcher also received 32 personal mails and participated regularly in BrickBuilders' activities: posting comments, asking questions, and receiving feedback from members, thus gaining sense of membership. During the research, there was an opportunity to visit the LEGO company with members of the community and the researcher attended the ModelExpo 2011 event, where BrickBuilders had a stand. Those events made it possible to speak with BrickBuilders' members personally, and 11 interviews were conducted.

For data analysis and iterative interpretation of findings, the researcher used manual analytic coding and hermeneutic interpretation (Bernard, 2004: tinyurl.com/ 7ltq5wm; Kozinets, 2006: tinyurl.com/7j92tjp; Kozinets, 2010: tinyurl.com/7eoklae; Moisander and Valtonen, 2006; tinyurl.com/cgrc4gn).

The researcher's subjective interpretation is a major challenge to netnography and the data analysis. The form of data is mostly textual. Moreover, the amount of data that can be found online is immense. In order to interpret the data correctly, the researcher must possess knowledge of that particular online culture (Kozinets, 2006; tinyurl.com/7j92tjp). 


\title{
Collective Value Creation and Empowerment in an Online Brand Community
}

\author{
Hanna Kurikko and Pekka Tuominen
}

In this study, the feelings and tone of the texts are being analyzed in the same way as words. The researcher tried to read beyond the postings to find the true meaning of the texts. Within a textual reality, the anonymity that is sometimes advantageous for obtaining disclosure prevents the researcher from having confidence that she understands the discloser (Kozinets, 2006). In this study the researcher met BrickBuilders' members and went places where community members had meetings. These face-to-face meetings helped increase the reliability of the interpretations.

\section{Main Features of BrickBuilders}

This study into BrickBuilders showed how the three features of brand communities and value co-creation (i.e., a sense of belonging, shared rituals and traditions, and a sense of moral responsibility) occur in practice and what motivates people to participate in an online brand community.

In BrickBuilders the sense of belonging, the most important element of brand communities (Muniz and O'Guinn, 2001; tinyurl.com/d73sov4), is based on shared enthusiasm for LEGO. BrickBuilders feel an important connection to LEGO, but at the same time they feel a strong connection toward like-minded people.

BrickBuilders offers a place for LEGO discussions, spreading LEGO related news, and sharing new ideas and opinions with like-minded people enabling collective value creation. This sense of belonging, or "weness", motivates members to join BrickBuilders (Heinonen and Halonen, 2007; tinyurl.com/cl8qwwb).

In BrickBuilders, support from the community is not limited to LEGO as a hobby. The members have learned organizational skills, cooperation, trust, and courage as well. This community has also influenced its members' identities, and contributions to identity have been identified as motivators for membership in online brand communities (Heinonen and Halonen, 2007: tinyurl.com/cl8qwwb; Bagozzi and Dholakia, 2006: tinyurl.com/7qftu4v).

BrickBuilders has shared rituals and traditions that perpetuate the community's shared history, culture, and consciousness and include certain behavioral norms and values (Muniz and O'Guinn, 2001; tinyurl.com/d73sov4). BrickBuilders' members are interested in LEGO's history and old narratives associated with the brand. Older members greet and assist new members in their brand learning and community so- cialization (Muniz and O'Guinn, 2001; Schau et al., 2009: tinyurl.com/bsh984y); they encourage new members to read the rules and instructions for the online forum, help others constantly, and take an active part in a variety of actions in the community.

A sense of moral responsibility (Muniz \& O'Guinn, 2001) is felt in BrickBuilders. The members have two missions: i) integrating and retaining members and ii) assisting other members in the proper use of LEGO products. Active members have a need to raise BrickBuilders' profile. The forum contains user-generated promotional material and new members are being actively recruited at events such as ModelExpo. The members help and advise others every day in the forum.

\section{Collective Value Creation in BrickBuilders}

The process of value co-creation consists of four thematic practices, namely social networking, impression management, community engagement, and brand use (Schau et al., 2009; tinyurl.com/bsh984y). Each of these practices can be identified in BrickBuilders.

Social networking is a practice that focuses on creating, enhancing, and sustaining ties among brand community members. BrickBuilders has been praised by new members for its positive welcoming and good overall team spirit, including governing the community by rules. BrickBuilders offers emotional and practical support to people sharing similar interests. It includes support for product-related issues such as customizing and creating new LEGO structures. The members are interested in receiving feedback (Heinonen and Halonen, 2007; tinyurl.com/cl8qwwb) from like-minded fans that truly understand the art of LEGO building. Members also support each other in non-brand-related life issues such as illness or bullying.

Impression management means that community members act as ambassadors of goodwill (Schau et al., 2009; tinyurl.com/bsh984y). However, in light of this study, the definition for "impression management" does not fully describe BrickBuilders' practices. Impression management in BrickBuilders can viewed as having internal and external components. This view should be a topic for further studies.

Internal impression management occurs inside the community. Members share news about LEGO in the forum, inspiring others to use the brand. One of BrickBuilders' active members is a LEGO Ambassador (tinyurl.com/86e5qns), who maintains communication 


\section{Collective Value Creation and Empowerment in an Online Brand Community}

Hanna Kurikko and Pekka Tuominen

between LEGO hobbyists and the LEGO company. Impression management may also involve negative comparisons with other competing brands and "pirate" bricks.

External impression management has two concrete factors. First, all writing in the forum creates impressions of BrickBuilders to all readers, both registered (private) and unregistered (public). Hence, correct language and good behaviour are essential. The second important factor is common events. Events are used, for example, to recruit new members and attract media attention.

Community-engagement practices reinforce members' commitment to the brand community. The members of BrickBuilders bring out brand experiences and confess openly that they are fans of a particular brand. For example, view the "Best in Show" video (tinyurl.com/7vfq65k) from a BrickBuilders exhibition. Members actively participate in community discussions, in events, and through self-motivated promotion efforts. The community is capable of organizing and building a large stand full of structures made of LEGO bricks. Activities are organized mainly via the BrickBuilders forum, the Skype communication system (skype.com), and the Doodle scheduling platform (doodle.com). Members travel great distances to take part in these events. After every event, feedback is collected about what went well and what kind of things they need to improve for future events; this is a form of collaborative learning.

Further collaborative learning can be related to LEGO products (i.e., building, creating) or to everyday skills such as cooperation, teamwork, and identity construction. Members share advice and ask for help from other members.

Brand use and development practices are specifically related to improved or enhanced use of the focal brand (Schau et al., 2009; tinyurl.com/bsh984y). Modifying the products and generating new ideas is one of the major activities in BrickBuilders. For example, one member developed a computer-based service called Basebrick (basebrick.com). This service helps LEGO fans to keep an inventory of the number and types of bricks that they own. The developer received help and advice from BrickBuilders' members during the development process. The ambassador project is another example of this type of activity. Advice and interactions between members also can be related to issues such as washing LEGO bricks or insuring a LEGO collection.
BrickBuilders' members develop and share novel ideas with other members and with the brand owner, relating to how the products could be developed (Schau et al., 2009). BrickBuilders' active members act as a liaison between LEGO hobbyists and the LEGO company. This kind of feedback is very valuable to the LEGO company.

\section{BrickBuilders' Collective Value Creation and Empowerment}

BrickBuilders' members feel a sense of belonging, they create value together, and they share similar motivations. The online brand community helps members to share their hobby and interests with congenial and likeminded people. Feedback and new ideas for improvement have been reported to the LEGO company via BrickBuilders. Thus, BrickBuilders can be considered to be a collectively creative and empowered online brand community.

The community's collective value creation and empowerment does not automatically lead to bidirectional communication with the company or other stakeholders. However, BrickBuilders' activity allows such cooperation in which both BrickBuilders and the LEGO company are willing to develop the relationship further. The company's investments in BrickBuilders are still limited. The community members would be motivated to have even more active collaboration with the LEGO company, which could pay more attention to this potential channel of marketing and cooperation.

\section{Conclusions}

Based on our research, we found evidence of intensive activity and positivity within an online community called BrickBuilders. The community members share their LEGO enthusiasm and mutual support. This sharing encourages the members to spread their skills and ideas actively throughout the community. A hobby of nearly professional LEGO building, which non-members may see as even slightly strange, further strengthens the bonds between members. The sense of belonging, strong motivation, and value co-creation are keys for empowerment.

An important note is that BrickBuilders is a fully fanbased community that is not affiliated with the LEGO company. Instead, BrickBuilders is born out of the fans' joint interest in the LEGO world. Being an open online community, the everyday conversations, events, and innovations organized and developed in BrickBuilders 


\section{Collective Value Creation and Empowerment in an Online Brand Community}

Hanna Kurikko and Pekka Tuominen

can be easily monitored by the LEGO company. The ideas being cultivated within the community could prove very significant to LEGO's research, development, and marketing. The potential benefits to the LEGO company are strengthened by the intense passion the members show in the LEGO world.

In general, companies and their stakeholders should use the experiences and ideas born within online communities. Companies already have access to large amount of data arising daily from these communities. This data should be used more actively and courageously. Companies should explore the communities and other online sources to learn how their actions, services, and products are being received. Co-operation between companies and active communities could prove to be surprisingly beneficial.

The target of the modern marketer is to enhance collective value creation between different stakeholders. A one-sided monologue is not sufficient. Brand communities allow mutual dialogue, further strengthening the community-company relationship and offering community members new opportunities. Online communities provide real and significant opportunities for marketing.

\section{About the Authors}

Hanna Kurikko holds an MSc (Econ.) in Marketing from the School of Management at the University of Tampere, Finland. Online brand communities, branding, and services are her main research interests.

Pekka Tuominen is Professor of Marketing at the University of Tampere, Finland. He is also a Docent at the University of Turku, Finland. His main research interests include strategic brand management, relationship marketing, and service marketing. He has attended several international conferences and his work has been published in many international journals.

Citation: Kurikko, H. and P. Tuominen. 2012. Collective

Value Creation and Empowerment in an Online Brand

Community: A Netnographic Study on LEGO Builders.

Technology Innovation Management Review. June 2012:

12-17. 